\title{
Exponential Stability in Mean Square for Neutral Stochastic Partial Functional Differential Equations with Impulses
}

\author{
Nan Ding \\ School of Mathematics and Statistics, Chongqing Three Gorges University, Wanzhou 404100, China \\ Correspondence should be addressed to Nan Ding; dingnan8118@126.com
}

Received 16 January 2013; Accepted 19 May 2013

Academic Editor: Vu Phat

Copyright (C) 2013 Nan Ding. This is an open access article distributed under the Creative Commons Attribution License, which permits unrestricted use, distribution, and reproduction in any medium, provided the original work is properly cited.

We discuss the exponential stability in mean square of mild solution for neutral stochastic partial functional differential equations with impulses. By applying impulsive Gronwall-Bellman inequality, the stochastic analytic techniques, the fractional power of operator, and semigroup theory, we obtain some completely new sufficient conditions ensuring the exponential stability in mean square of mild solution for neutral stochastic partial functional differential equations with impulses. Finally, an example is provided to illustrate the obtained theory.

\section{Introduction}

Stochastic partial differential equations have attracted the attention of many authors, and many valuable results on the existence, uniqueness, and stability of mild solution have been established. For example, Ren and Sakthivel [1] have established the existence and uniqueness of mild solution for a class of second-order neutral stochastic evolution equations with infinite delay and Poisson jumps by means of the successive approximation and the continuous dependence of solutions on the initial data by means of a corollary of the Bihari inequality; Sakthivel et al. [2,3] have discussed the existence and uniqueness of square-mean pseudo almost automorphic mild solutions for stochastic fractional differential equations by using the stochastic analysis theory, fixed point strategy, and the existence of mild solution to nonlinear stochastic fractional differential by using fractional calculations, fixed point technique, stochastic analysis theory and methods adopted directed from deterministic fractional equations, respectively; Sakthivel et al. $[4,5]$ also have derived the exponential stability of mild solutions to the second-order stochastic evolution equations with Poisson jumps by applying stochastic analysis theory, and the existence and asymptotic stability in pth moment of mild solution to secondorder nonlinear neural stochastic differential equations with the help of fixed point theory, stochastic analysis technique, and semigroup theory, respectively. Besides stochastic effects, impulsive effects likewise exist in real-world models. It is to be noted that there has been increasing interest in the study of the existence, uniqueness, and stability of mild solution for stochastic partial functional differential equations with impulses due to its wide applications in various sciences, and many significant results have been obtained [6-15].

To the best of the author's knowledge, there are only few works about the exponential stability of mild solution to neutral stochastic partial functional differential equations with impulses. One of the reasons is that the mild solutions do not have stochastic differentials, so Itô formula fails to deal with this problem. Another reason is that when we consider the exponential stability of mild solution for stochastic partial functional differential equations with impulses, impulsive effects on the system brings about many difficulties, since the corresponding theory for such problem has not yet been fully developed. For example, Sakthivel and Luo $[8,9]$ have discussed the asymptotic stability for mild solution of impulsive stochastic partial differential equations by using the fixed point theorem which can be regarded as an excellent tool to derive the exponential stability for mild solution to stochastic partial differential with delays in Luo $[10,11]$; this very useful method may be difficult and even ineffective for the exponential stability of such system with impulses; some other methods used in Caraballo and Liu [12], 
Wan and Duan [13], and so forth are also ineffective for this problem, since mild solutions do not have stochastic differentials; in addition, Chen [14] established an impulsiveintegral inequality to investigate the exponential stability of stochastic partial differential equation with delays and impulses, but it is not effective for neutral type. For the previous reasons, Long et al. [15] established a new impulsiveintegral inequality to investigate the global attracting set and exponential $p$-stability of stochastic neutral partial functional differential equations with impulses. However, the methods of studying the exponential stability of mild solution for neutral stochastic partial functional differential equations with impulses is still not abundant.

Motivated by the previous discussion, in this paper, we discuss exponential stability in mean square of mild solution for neutral stochastic partial functional differential equations with impulses. By applying impulsive Gronwall-Bellman inequality, the stochastic analytic techniques, inequality technique, the fractional power of operator, and semigroup theory, we obtain some completely new sufficient conditions to ensure the exponential stability in mean square of mild solution for stochastic partial functional differential equations with impulses.

The rest of this paper is organized as follows. In Section 2, we present some basic notations, definitions, and auxiliary results. In Section 3, sufficient conditions are derived to ensure the exponential stability in mean square for mild solution. Finally, an example is given to demonstrate the obtained results.

\section{Preliminaries}

Let $\left(\Omega, \mathscr{F},\{\mathscr{F}\}_{t \geq 0}, \mathbb{P}\right)$ be a complete probability space with a filtration $\{\mathscr{F}\}_{t \geq 0}$ satisfying the usual conditions (i.e., it is right continuous and $\mathscr{F}_{0}$ contains all $\mathbb{P}$-null sets).

Let $H, K$ be real separable Hilbert spaces, let and $\mathscr{L}(K, H)$ be the space of bounded linear operators mapping $K$ into $H$. For convenience, we will use the same notations $\|\cdot\|$ to denote the norms in $H, K$, and $\mathscr{L}(K, H)$ without any confusion. Let $\{\omega(t): t \geq 0\}$ denotes a $K$-valued $\{\mathscr{F}\}_{t \geq 0}$-Wiener process defined on $\left(\Omega, \mathscr{F},\{\mathscr{F}\}_{t \geq 0}, \mathbb{P}\right)$ with covariance operator $Q$; that is,

$$
\mathrm{E}\langle\omega(t), x\rangle_{K}\langle\omega(s), y\rangle_{K}=(t \wedge s)\langle Q x, y\rangle_{K}, \quad x, y \in K
$$

where $Q$ is a positive self-adjoint, trace class operator on $K$, $\langle\cdot, \cdot\rangle_{K}$ denotes the inner product of $K$, and $E$ denotes the mathematical expectation. In particular, we call such $\omega(t)$ : $t \geq 0$, a $K$-valued $Q$-Wiener process with respect to $\{\mathscr{F}\}_{t \geq 0}$.

In order to define stochastic integrals with respect to the Q-Wiener process $\omega(t)$, we introduce the subspace $K_{0}=$ $Q^{1 / 2}(K)$ of $K$ which, endowed with the inner product $\langle u, v\rangle_{K_{0}}=\left\langle Q^{-1 / 2} u, Q^{-1 / 2} v\right\rangle_{K}$, is a Hilbert space. We assume that there exists a complete orthonormal system $\left\{e_{i}\right\}_{i \geq 1}$ in $K$, a bounded sequence of nonnegative real numbers $\lambda_{i}$ such that $Q e_{i}=\lambda_{i} e_{i}, i=1,2, \ldots$, and a sequence $\left\{\beta_{i}(t)\right\}_{i \geq 1}$ of independent Brownian motions such that

$$
\langle\omega(t), e\rangle=\sum_{i=1}^{\infty} \sqrt{\lambda_{i}}\left\langle e_{i}, e\right\rangle \beta_{i}(t), \quad e \in K
$$

and $B_{t}=B_{t}^{\omega}$, where $B_{t}^{\omega}$ is the sigma algebra generated by $\{\omega(s): 0 \leq s \leq t\}$. Let $\mathscr{L}_{2}^{0}=\mathscr{L}_{2}\left(K_{0}, H\right)$ denote the space of all Hilbert-Schmidt operators from $K_{0}$ into $H$. It turns out to be a separable Hilbert space, equipped with the norm

$$
\|\psi\|_{\mathscr{L}_{0}^{2}}^{2}=\operatorname{tr}\left(\left(\psi Q^{1 / 2}\right)\left(\psi Q^{1 / 2}\right)^{*}\right) \quad \text { for any } \psi \in \mathscr{L}_{2}^{0} \text {. }
$$

Clearly, for any bounded operators $\psi \in \mathscr{L}(K, H)$, this norm reduces to $\|\psi\|_{\mathscr{L}_{2}^{0}}^{2}=\operatorname{tr}\left(\psi Q \psi^{*}\right)$.

Let $\mathbb{R}$ and $\mathbb{Z}$ be the sets of real and integer numbers, respectively; let $\mathbb{R}^{+}=[0,+\infty)$ and $C(X, Y)$ denote the space of continuous mapping from the topological space $X$ to the topological space $Y$. Particularly, $C \triangleq C([-\tau, 0], \mathbb{R})$ denotes the family of all continuous $\mathbb{R}$-valued functions $\phi$ defined on $[-\tau, 0]$ with the norm $\|\phi\|_{\tau}=\sup _{-\tau \leq \theta \leq 0}\|\phi(\theta)\|$, where $\tau$ is a positive constant.

$\operatorname{PC}\left(J, \mathbb{R}^{n}\right)=\left\{\varphi: J \rightarrow \mathbb{R}^{n}\right.$ is continuous for all, but at most a finite number of points $t \in J$ and at these points $t \in J$, $\varphi\left(t^{+}\right)$and $\varphi\left(t^{-}\right)$exist, $\left.\varphi\left(t^{+}\right)=\varphi(t)\right\}$, where $J \subset \mathbb{R}$ is a bounded interval and $\varphi\left(t^{+}\right)$and $\varphi\left(t^{-}\right)$denote the right-hand and lefthand limits of the function $\varphi(t)$, respectively. Particularly, let $\mathrm{PC} \triangleq \mathrm{PC}([-\tau, 0], H)$.

Let $\mathrm{PC}_{\mathscr{F}_{0}}^{b}([-\tau, 0], H)\left(\mathrm{PC}_{\mathscr{F}_{t}}^{b}([-\tau, 0], H)\right)$ denote the family of all bounded $\mathscr{F}_{0}\left(\mathscr{F}_{t}\right)$-measurable, $\operatorname{PC}([-\tau, 0], H)$-valued random variables $\phi$, satisfying $\|\phi\|_{\mathscr{L}_{2}}^{2}=\sup _{-\tau \leq \theta \leq 0} \mathrm{E}\|\phi(\theta)\|_{H}^{2}$.

In this paper, we consider the following neutral stochastic partial functional differential equation with impulses:

$$
\begin{aligned}
& d[x(t)+g(t, x(t-\tau))] \\
& =[A x(t)+f(t, x(t-\tau))] d t \\
& +\sigma(t, x(t-\tau)) d \omega(t), \quad t \geq 0, t \neq t_{k}, \\
& \Delta x\left(t_{k}\right)=x\left(t_{k}^{+}\right)-x\left(t_{k}^{-}\right) \\
& =I_{k}\left(x\left(t_{k}\right)\right), \quad t=t_{k}, k \in \mathbb{Z}, \\
& x_{0}(s)=\phi(s) \in \mathrm{PC}_{\mathscr{F}_{0}}^{b}([-\tau, 0], H), \quad s \in[-\tau, 0] \text {, a.s, }
\end{aligned}
$$

where $A: D(A) \subset H \rightarrow H$ is the infinitesimal generator of an analytic semigroup of linear operator $S(t)_{t \geq 0}$ on a Hilbert space $H ; f, g: \mathbb{R}^{+} \times H \rightarrow H$ and $\sigma: \mathbb{R}^{+} \times H \rightarrow \mathscr{L}_{2}^{0}$ are jointly continuous functions; the fixed moment of time $t_{k}$ satisfies $0<t_{1}<t_{2}<\cdots<t_{k}<\cdots$, and $\lim _{k \rightarrow+\infty}=$ $+\infty ; x\left(t_{k}^{+}\right)$and $x\left(t_{k}^{-}\right)$represent the right and left limits of $x(t)$ at $t=t_{k}, k=1,2, \ldots$, respectively; $\Delta x\left(t_{k}\right)=x\left(t_{k}^{+}\right)-$ $x\left(t_{k}^{-}\right)$represents the jump in the state $x$ at time $t_{k}$ with $I_{k}$ determining the size of the jump.

We also assume that $0 \in \rho(-A)$, the resolvent set of $-A$. Then, it is possible to define the fractional power $(-A)^{\alpha}$ for some $0<\alpha \leq 1$ as a closed linear operator with its domain 
$D\left((-A)^{\alpha}\right)$; furthermore, the subspace $D\left((-A)^{\alpha}\right)$ is dense in $H$, and the expression

$$
\|x\|_{\alpha}=\left\|(-A)^{\alpha} x\right\|_{H}, \quad x \in D\left((-A)^{\alpha}\right),
$$

defines a norm on $D\left((-A)^{\alpha}\right)$.

Lemma 1 (see Pazy [16]). Suppose that $0 \in \rho(-A)$, then, we know that there exist constants $M \geq 1, \gamma>0$ such that $\|S(t)\| \leq$ $M e^{-\gamma t}$ for $t \geq 0$, and for every $0<\alpha \leq 1$

(i) we have for each $x \in D\left((-A)^{\alpha}\right)$,

$$
S(t)(-A)^{\alpha} x=(-A)^{\alpha} S(t) x ;
$$

(ii) there exists $M_{\alpha}>0$ such that

$$
\left\|(-A)^{\alpha} S(t)\right\| \leq M_{\alpha} t^{-\alpha} e^{-\gamma t} .
$$

Definition 2. A stochastic process $x(t), t \in \mathbb{R}^{+}$, is called a mild solution of the system (4), if

(i) $x(t)$ is an $\mathscr{F}_{t}(t \geq 0)$ adapted process,

(ii) $x(t) \in H$ has a càdlàg path on $t \in \mathbb{R}^{+}$almost surely,

(iii) for arbitrary $t \in \mathbb{R}^{+}$, we have

$$
\begin{aligned}
x(t)= & S(t)[\phi(0)+g(0, \phi)]-g(t, x(t-\tau)) \\
& -\int_{0}^{t} A S(t-s) g(s, x(s-\tau)) d s \\
& +\int_{0}^{t} S(t-s) f(s, x(s-\tau)) d s \\
& +\int_{0}^{t} S(t-s) \sigma(s, x(s-\tau)) d \omega(s) \\
& +\sum_{0<t_{k}<t} S\left(t-t_{k}\right) I_{k}\left(x\left(t_{k}\right)\right),
\end{aligned}
$$

where $x_{0}(\cdot) \in \mathrm{PC}_{\mathscr{F}_{0}}^{b}([-\tau, 0], H)$, a.s.

Definition 3. The mild solution of system (4) is said to be exponentially stable in mean square if there exists a pair of positive constants $\lambda>0$ and $M \geq 1$ such that for any solution $x(t)$ with the initial condition $\phi \in \mathrm{PC}_{\mathscr{F}_{0}}^{b}([-\tau, 0], H)$,

$$
\mathrm{E}\|x(t)\|^{2} \leq M\|\phi\|_{\mathscr{L}_{2}}^{2} e^{-\lambda t}, \quad t \geq 0 .
$$

\section{Exponential Stability}

For system (4), we impose the following assumptions.

(A1) There exist constants $L_{f}>0, L_{\sigma}>0$ such that for any $x, y \in H$ and $t \geq 0$,

$$
\begin{aligned}
\|f(t, x)-f(t, y)\| \leq L_{f}\|x-y\|, & f(t, 0)=0, \\
\|\sigma(t, x)-\sigma(t, y)\|_{\mathscr{L}_{2}^{0}} \leq L_{\sigma}\|x-y\|, & \sigma(t, 0)=0 .
\end{aligned}
$$

(A2) There exist $\alpha \in(0,1], L_{g}>0$ such that for any $x, y \in$ $H$ and $t \geq 0$,

$$
\begin{gathered}
g(t, x) \in D\left((-A)^{\alpha}\right), \\
\left\|(-A)^{\alpha} g(t, x)-(-A)^{\alpha} g(t, y)\right\| \leq L_{g}\|x-y\|, \\
g(t, 0)=0 .
\end{gathered}
$$

(A3) There exist some positive numbers $q_{k}(k=1,2, \ldots)$ such that

$$
\left\|I_{k}(x)-I_{k}(y)\right\| \leq q_{k}\|x-y\|, \quad I_{k}(0)=0, k=1,2, \ldots,
$$

for any $x, y \in H$ and $\sum_{k=1}^{\infty} q_{k}<\infty$.

Under the assumptions, (A1)-(A3), the existence and uniqueness of mild solution to the system (4) are easily shown by using Picard iterative method.

Theorem 4. Suppose that the assumptions (A1)-(A3) hold; furthermore, and the following assumptions

$$
\begin{aligned}
& \text { (A4) } \inf _{k=1,2, \ldots}\left(t_{k}-t_{k-1}\right)=\theta>0, \\
& \begin{array}{l}
\text { (A5) } 0<\left(\left(18 M_{1-\alpha}^{2} L_{g}^{2} \gamma^{1-2 \alpha} \Gamma(2 \alpha-1)+3 M^{2} L_{f}^{2} \gamma^{-1}+\right.\right. \\
\left.\left.3 M^{2} L_{\sigma}^{2}\right) e^{\gamma \tau}+(1 / \theta) \ln \left(6 M^{2}\left(\sum_{k=1}^{\infty} q_{k}\right)^{2}\right)\right) /(1- \\
\left.18\left\|(-A)^{-\alpha}\right\|^{2} L_{g}^{2} e^{\gamma \tau}\right)<\gamma
\end{array}
\end{aligned}
$$

hold for $\alpha \in(1 / 2,1]$, where $\Gamma(\cdot)$ is the Gamma function and $M, M_{1-\alpha}$, and $\gamma$ are corresponding constants in Lemma 1 . Then the mild solution of system (4) is exponentially stable in mean square.

Proof. From (8), for any $t \geq 0$, we can get

$$
\begin{aligned}
\mathrm{E}\|x(t)\|^{2}=\mathrm{E} \| S(t)[\phi(0)+g(0, \phi)]-g(t, x(t-\tau)) \\
\quad-\int_{0}^{t} A S(t-s) g(s, x(s-\tau)) d s \\
+\int_{0}^{t} S(t-s) f(s, x(s-\tau)) d s \\
+\int_{0}^{t} S(t-s) \sigma(s, x(s-\tau)) d \omega(s) \\
+\sum_{0<t_{k}<t} S\left(t-t_{k}\right) I_{k}\left(x\left(t_{k}\right)\right) \|^{2}
\end{aligned}
$$




$$
\begin{aligned}
& \leq 3 \mathrm{E} \| S(t)[\phi(0)+g(0, \phi)]-g(t, x(t-\tau)) \\
& \quad-\int_{0}^{t} A S(t-s) g(s, x(s-\tau)) d s \\
& +\sum_{0<t_{k}<t} S\left(t-t_{k}\right) I_{k}\left(x\left(t_{k}\right)\right) \|^{2} \\
& +3 \mathrm{E}\left\|\int_{0}^{t} S(t-s) f(s, x(s-\tau)) d s\right\|^{2} \\
& +3 \mathrm{E}\left\|\int_{0}^{t} S(t-s) \sigma(s, x(s-\tau)) d \omega(s)\right\|^{2} \\
& +18 \mathrm{E}\|S(t)[\phi(0)+g(0, \phi)]\|^{2} \\
& +18 \mathrm{E}\|g(t, x(t-\tau))\|^{2} \\
& +3 \mathrm{E}\left\|\int_{i}^{t} S(t-s) f(s, x(s-\tau)) d s\right\|^{2} S(t-s) \sigma(s, x(s-\tau)) d \omega(s) \|^{2} \\
& +18 \mathrm{E}\left\|\int_{0}^{t}-A S(t-s) g(s, x(s-\tau)) d s\right\|^{2} \\
& +\sum_{0<t_{k}<t} S\left(t-t_{k}\right) I_{k}\left(x\left(t_{k}\right)\right)\|\|^{2}
\end{aligned}
$$

It follows from (A2) that

$$
\begin{aligned}
J_{1}= & 18 \mathrm{E}\|S(t)[\phi(0)+g(0, \phi)]\|^{2} \\
\leq & 18\|S(t)\|^{2} \\
& \times \mathrm{E}\left(\|\phi(0)\|+\left\|(-A)^{-\alpha}\right\|\left\|(-A)^{\alpha} g(0, \phi)\right\|\right)^{2} \\
\leq & 18 M^{2} e^{-2 \gamma t} \mathrm{E}\left(\|\phi\|+\left\|(-A)^{-\alpha}\right\| L_{g}\|\phi\|\right)^{2} \\
\leq & 18 M^{2} e^{-\gamma t}\left(1+\left\|(-A)^{-\alpha}\right\| L_{g}\right)^{2}\|\phi\|_{\mathscr{L}_{2}}^{2} .
\end{aligned}
$$

By (A2), we can get

$$
\begin{aligned}
J_{2} & =18 \mathrm{E}\|g(t, x(t-\tau))\|^{2} \\
& =18 \mathrm{E}\left\|(-A)^{-\alpha}(-A)^{\alpha} g(t, x(t-\tau))\right\|^{2} \\
& \leq 18\left\|(-A)^{-\alpha}\right\|^{2} L_{g}^{2} \mathrm{E}\|x(t-\tau)\|^{2} .
\end{aligned}
$$

Employing Lemma 1, (A2), and Hölder inequality, we obtain

$$
\begin{aligned}
J_{3}= & 18 \mathrm{E}\left\|\int_{0}^{t}-A S(t-s) g(s, x(s-\tau)) d s\right\|^{2} \\
\leq & 18 \mathrm{E}\left(\int_{0}^{t}\left\|(-A)^{1-\alpha} S(t-s)\right\|\right. \\
\leq & 18 M_{1-\alpha}^{2} L_{g}^{2} \mathrm{E} \\
& \left.\times\left(\int_{0}^{t} e^{-\gamma(t-s)}(t-s)^{\alpha-1}\|x(s-\tau)\| d s\right)^{2} g(s, x(s-\tau)) \| d s\right)^{2} \\
\leq & 18 M_{1-\alpha}^{2} L_{g}^{2}\left(\int_{0}^{t} e^{-\gamma(t-s)}(t-s)^{2(\alpha-1)} d s\right) \\
\leq & \quad 18 M_{1-\alpha}^{2} L_{g}^{2} \gamma^{1-2 \alpha} \Gamma(2 \alpha-1) \\
& \times\left(\int_{0}^{t} e^{-\gamma(t-s)} \mathrm{E}\|x(s-\tau)\|^{2} d s\right) \\
\leq & 18 M_{1-\alpha}^{2} L_{g}^{2} \gamma^{1-2 \alpha} \Gamma(2 \alpha-1) \\
& \times \int_{-\tau}^{t-\tau} e^{\gamma \tau} e^{-\gamma(t-s)} \mathrm{E}\|x(s)\|^{2} d s
\end{aligned}
$$

Combining Lemma 1 and (A3), with Hölder inequality, we can get

$$
\begin{aligned}
J_{4} & =6 \mathrm{E}\left\|\sum_{0<t_{k}<t} S\left(t-t_{k}\right) I_{k}\left(x\left(t_{k}\right)\right)\right\|^{2} \\
& \leq 6 \mathrm{E}\left(\sum_{0<t_{k}<t}\left\|S\left(t-t_{k}\right)\right\|\left\|I_{k}\left(x\left(t_{k}\right)\right)\right\|\right)^{2} \\
& \leq 6 M^{2} \mathrm{E}\left(\sum_{0<t_{k}<t} e^{-\gamma\left(t-t_{k}\right)} q_{k}\left\|x\left(t_{k}\right)\right\|\right)^{2}
\end{aligned}
$$

$$
\begin{aligned}
& \leq 6 M^{2}\left(\sum_{k=1}^{\infty} q_{k}\right)\left(\sum_{0<t_{k}<t} e^{-\gamma\left(t-t_{k}\right)} q_{k} \mathrm{E}\left\|x\left(t_{k}\right)\right\|^{2}\right) \\
& \leq 6 M^{2}\left(\sum_{k=1}^{\infty} q_{k}\right)^{2}\left(\sum_{0<t_{k}<t} e^{-\gamma\left(t-t_{k}\right)} \mathrm{E}\left\|x\left(t_{k}\right)\right\|^{2}\right) .
\end{aligned}
$$


From Lemma 1, (A1) and Hölder inequality, we obtain

$$
\begin{aligned}
J_{5}= & 3 \mathrm{E}\left\|\int_{0}^{t} S(t-s) f(s, x(s-\tau)) d s\right\|^{2} \\
\leq & 3 \mathrm{E}\left(\int_{0}^{t} \|(S(t-s)\|\| f(s, x(s-\tau)) \| d s)^{2}\right. \\
\leq & 3 M^{2} L_{f}^{2} \mathrm{E}\left(\int_{0}^{t} e^{-\gamma(t-s)}\|x(s-\tau)\| d s\right)^{2} \\
\leq & 3 M^{2} L_{f}^{2}\left(\int_{0}^{t} e^{-\gamma(t-s)} d s\right) \\
& \times\left(\int_{0}^{t} e^{-\gamma(t-s)} \mathrm{E}\|x(s-\tau)\|^{2} d s\right) \\
\leq & 3 M^{2} L_{f}^{2} \gamma^{-1}\left[\gamma^{-1} e^{-\gamma t}\left(e^{\gamma \tau}-1\right)\|\phi\|_{\mathscr{L}_{2}}^{2}\right. \\
& \left.+e^{\gamma \tau} \int_{0}^{t-\tau} e^{-\gamma(t-s)} \mathrm{E}\|x(s)\|^{2} d s\right] .
\end{aligned}
$$

Using (A1) and Burkholder-type inequality, we obtain

$$
\begin{aligned}
J_{6} & =3 \mathrm{E}\left\|\int_{0}^{t} S(t-s) \sigma(s, x(s-\tau)) d \omega(s)\right\|^{2} \\
& \leq 3 \mathrm{E} \int_{0}^{t}\|S(t-s)\|^{2}\|\sigma(s, x(s-\tau))\|_{\mathscr{L}_{2}^{0}}^{2} d s \\
\leq & 3 M^{2} L_{\sigma}^{2} \int_{0}^{t} e^{-2 \gamma(t-s)} \mathrm{E}\|x(s-\tau)\|^{2} d s \\
\leq & 3 M^{2} L_{\sigma}^{2}\left[\gamma^{-1} e^{-\gamma t}\left(e^{\gamma \tau}-1\right)\|\phi\|_{\mathscr{L}_{2}}^{2}\right. \\
& \left.+e^{\gamma \tau} \int_{0}^{t-\tau} e^{-\gamma(t-s)} \mathrm{E}\|x(s)\|^{2} d s\right] .
\end{aligned}
$$

Substituting (14)-(19) into (13), we have

$$
\begin{aligned}
\mathrm{E}\|x(t)\|^{2} e^{\gamma t} \leq & 18 M^{2}\left(1+\left\|(-A)^{-\alpha}\right\| L_{g}\right)^{2}\|\phi\|_{\mathscr{L}_{2}}^{2} \\
& +18\left\|(-A)^{-\alpha}\right\|^{2} L_{g}^{2} \mathrm{E}_{\{t-\tau<0\}}\|x(t-\tau)\|^{2} e^{\gamma t} \\
& +18\left\|(-A)^{-\alpha}\right\|^{2} L_{g}^{2} \\
& \times \mathrm{E}_{\{t-\tau \geq 0\}}\|x(t-\tau)\|^{2} e^{\gamma t} \\
& +18 M_{1-\alpha}^{2} L_{g}^{2} \gamma^{1-2 \alpha} \Gamma(2 \alpha-1) \\
& \times\left[\gamma^{-1}\left(e^{\gamma \tau}-1\right)\|\phi\|_{\mathscr{L}_{2}}^{2}+e^{\gamma \tau} \int_{0}^{t} \mathrm{E}\|x(s)\|^{2} e^{\gamma s} d s\right]
\end{aligned}
$$

$$
\begin{aligned}
& +6 M^{2}\left(\sum_{k=1}^{\infty} q_{k}\right)^{2}\left(\sum_{0<t_{k}<t} \mathrm{E}\left\|x\left(t_{k}\right)\right\|^{2} e^{\gamma t_{k}}\right) \\
& +3 M^{2} L_{f}^{2} \gamma^{-1} \\
& \times\left[\gamma^{-1}\left(e^{\gamma \tau}-1\right)\|\phi\|_{\mathscr{L}_{2}}^{2}+e^{\gamma \tau} \int_{0}^{t} \mathrm{E}\|x(s)\|^{2} e^{\gamma s} d s\right] \\
& +3 M^{2} L_{\sigma}^{2} \\
& \times\left[\gamma^{-1}\left(e^{\gamma \tau}-1\right)\|\phi\|_{\mathscr{L}_{2}}^{2}+e^{\gamma \tau} \int_{0}^{t} \mathrm{E}\|x(s)\|^{2} e^{\gamma s} d s\right] \\
& \leq 18 M^{2}\left(1+\left\|(-A)^{-\alpha}\right\| L_{g}\right)^{2}\|\phi\|_{\mathscr{L}_{2}}^{2} \\
& +18\left\|(-A)^{-\alpha}\right\|^{2} L_{g}^{2} e^{\gamma \tau}\|\phi\|_{\mathscr{L}_{2}}^{2} \\
& +18\left\|(-A)^{-\alpha}\right\|^{2} L_{g}^{2} e^{\gamma \tau} \mathrm{E}\|x(t)\|^{2} e^{\gamma t} \\
& +18 M_{1-\alpha}^{2} L_{g}^{2} \gamma^{-2 \alpha} \Gamma(2 \alpha-1)\left(e^{\gamma \tau}-1\right)\|\phi\|_{\mathscr{L}_{2}}^{2} \\
& +18 M_{1-\alpha}^{2} L_{g}^{2} \gamma^{1-2 \alpha} \Gamma(2 \alpha-1) e^{\gamma \tau} \\
& \times \int_{0}^{t} \mathrm{E}\|x(s)\|^{2} e^{\gamma s} d s \\
& +6 M^{2}\left(\sum_{k=1}^{\infty} q_{k}\right)^{2}\left(\sum_{0<t_{k}<t} \mathrm{E}\left\|x\left(t_{k}\right)\right\|^{2} e^{\gamma t_{k}}\right) \\
& +3 M^{2} L_{f}^{2} \gamma^{-2}\left(e^{\gamma \tau}-1\right)\|\phi\|_{\mathscr{L}_{2}}^{2} \\
& +3 M^{2} L_{f}^{2} \gamma^{-1} e^{\gamma \tau} \int_{0}^{t} \mathrm{E}\|x(s)\|^{2} e^{\gamma s} d s \\
& +3 M^{2} L_{\sigma}^{2} \gamma^{-1}\left(e^{\gamma \tau}-1\right)\|\phi\|_{\mathscr{L}_{2}}^{2} \\
& +3 M^{2} L_{\sigma}^{2} e^{\gamma \tau} \int_{0}^{t} \mathrm{E}\|x(s)\|^{2} e^{\gamma s} d s .
\end{aligned}
$$

From assumption (A5), we can get

$$
\begin{aligned}
\mathrm{E}\|x(t)\|^{2} e^{\lambda t} \leq & C\|\phi\|_{\mathscr{L}_{2}}^{2}+p \int_{0}^{t} \mathrm{E}\|x(s)\|^{2} e^{\gamma s} d s \\
& +\beta \sum_{0<t_{k}<t} \mathrm{E}\left\|x\left(t_{k}\right)\right\|^{2} e^{\gamma t_{k}},
\end{aligned}
$$

where $C \triangleq\left(1-18\left\|(-A)^{-\alpha}\right\|^{2} L_{g}^{2} e^{\gamma \tau}\right)^{-1}\left[18 M^{2}(1+\right.$ $\left.\left\|(-A)^{-\alpha}\right\| L_{g}\right)^{2}+18\left\|(-A)^{-\alpha}\right\|^{2} L_{g}^{2} e^{\gamma \tau}+18 M_{1-\alpha}^{2} L_{g}^{2} \gamma^{-2 \alpha} \Gamma(2 \alpha-$ 1) $\left.\left(e^{\gamma \tau}-1\right)+3 M^{2} L_{f}^{2} \gamma^{-2}\left(e^{\gamma \tau}-1\right)+3 M^{2} L_{\sigma}^{2} \gamma^{-1}\left(e^{\gamma \tau}-1\right)\right]$, $p \triangleq\left(1-18\left\|(-A)^{-\alpha}\right\|^{2} L_{g}^{2} e^{\gamma \tau}\right)^{-1}\left[18 M_{1-\alpha}^{2} L_{g}^{2} \gamma^{1-2 \alpha} \Gamma(2 \alpha-1) e^{\gamma \tau}+\right.$ $\left.3 M^{2} L_{f}^{2} \gamma^{-1} e^{\gamma \tau}+3 M^{2} L_{\sigma}^{2} e^{\gamma \tau}\right]$, and $\beta \triangleq \quad(1-$ $\left.18\left\|(-A)^{-\alpha}\right\|^{2} L_{g}^{2} e^{\gamma \tau}\right)^{-1} 6 M^{2}\left(\sum_{k=1}^{\infty} q_{k}\right)^{2}$.

According to Gronwall-Bellmen's inequality [17], we have

$$
\mathrm{E}\|x(t)\|^{2} \leq C\|\phi\|_{\mathscr{L}_{2}}^{2} \prod_{0<t_{k}<t}(1+\beta) e^{-(\gamma-p) t} .
$$


On the other hand, by (A4), one has

$$
\prod_{0<t_{k}<t}(1+\beta) \leq(1+\beta)^{t / \theta}=e^{(t / \theta) \ln (1+\beta)} .
$$

Thereby, (22) can be rewritten as

$$
\mathrm{E}\|x(t)\|^{2} \leq C\|\phi\|_{\mathscr{L}_{2}}^{2} \prod_{0<t_{k}<t}(1+\beta) e^{-(\gamma-p-\ln (1+\beta) / \theta) t},
$$

which implies the mild solution of system (4) is exponentially stable in mean square.

This completes the proof.

Theorem 5. Suppose that all the conditions of Theorem 4 hold. Then the mild solution of system (4) is exponential stable almost surely.

Proof. The proof is quite similar to the proof of Theorem 5.1 in [18], and we omit it here.

If the impulsive effects, $I_{k}(\cdot) \equiv 0(k=1,2, \ldots)$, system (4) is turned into the following neutral stochastic partial functional differential equations:

$$
\begin{aligned}
& d[x(t)+g(t, x(t-\tau))]= {[A x(t)+f(t, x(t-\tau))] d t } \\
&+\sigma(t, x(t-\tau)) d \omega(t), \quad t \geq 0, \\
& x_{0}(s)=\phi(s) \in C_{\mathscr{F}_{0}}^{b}([-\tau, 0], H), \quad s \in[-\tau, 0], \text { a.s. }
\end{aligned}
$$

Corollary 6. Assume that (A1)-(A2) hold, and the following condition

$$
\begin{aligned}
0 & <\frac{\left(9 M_{1-\alpha}^{2} L_{g}^{2} \gamma^{1-2 \alpha} \Gamma(2 \alpha-1)+3 M^{2} L_{f}^{2} \gamma^{-1}+3 M^{2} L_{\sigma}^{2}\right) e^{\gamma \tau}}{1-9\left\|(-A)^{-\alpha}\right\|^{2} L_{g}^{2} e^{\gamma \tau}} \\
& <\gamma
\end{aligned}
$$

holds for $\alpha \in(1 / 2,1]$. Then the mild solution of system (25) is exponentially stable in mean square.

If $g(t, \cdot) \equiv 0$, then system (4) becomes stochastic partial functional differential equations with impulses as follows:

$$
\begin{gathered}
d x(t)=[A x(t)+f(t, x(t-\tau))] d t \\
\quad+\sigma(t, x(t-\tau)) d \omega(t), \quad t \geq 0, t \neq t_{k}, \\
\Delta x\left(t_{k}\right)=x\left(t_{k}^{+}\right)-x\left(t_{k}^{-}\right)=I_{k}\left(x\left(t_{k}\right)\right), \quad t=t_{k}, \quad k \in \mathbb{Z}, \\
x_{0}(s)=\phi(s) \in \mathrm{PC}_{\mathscr{F}_{0}}^{b}([-\tau, 0], H), \quad s \in[-\tau, 0], \text { a.s. }
\end{gathered}
$$

Corollary 7. Assume that (A1) and (A3) hold, and the following conditions

(A4) $\inf _{k=1,2, \ldots}\left(t_{k}-t_{k-1}\right)=\theta>0$,

(A5) $\left(3 M^{2} L_{f}^{2} \gamma^{-1}+3 M^{2} L_{\sigma}^{2}\right) e^{\gamma \tau}+(1 / \theta) \ln \left(6 M^{2}\left(\sum_{k=1}^{\infty} q_{k}\right)^{2}\right)<$ $\gamma$ hold. Then the mild solution of system (27) is exponentially stable in mean square.

If $g(t, \cdot) \equiv 0$ and $I_{k}(\cdot) \equiv 0$, then system (4) becomes stochastic partial functional differential equations as follows:

$$
\begin{gathered}
d x(t)=[A x(t)+f(t, x(t-\tau))] d t \\
+\sigma(t, x(t-\tau)) d \omega(t), \quad t \geq 0, \\
x_{0}(s)=\phi(s) \in C_{\mathscr{F}_{0}}^{b}([-\tau, 0], H), \quad s \in[-\tau, 0], \text { a.s. }
\end{gathered}
$$

Corollary 8. Assume that (A1) holds, and the following condition

$$
\left(3 M^{2} L_{f}^{2} \gamma^{-1}+3 M^{2} L_{\sigma}^{2}\right) e^{\gamma \tau}<\gamma
$$

holds. Then the mild solution of system (28) is exponentially stable in mean square.

Remark 9. In [12], Caraballo and Liu have studied the exponential stability in $p(p \geq 2)$-moment of mild solution to $(28)$ by utilizing Gronwall inequality, and the monotone decreasing behaviors of the delays are imposed. Particularly, when $\tau(t) \equiv \tau, \delta(t) \equiv \tau$, the condition ensuring the exponential stability in mean square is $\left(3 M^{2} L_{f}^{2} \gamma^{-1}+3 M^{2} L_{\sigma}^{2}\right) e^{\gamma \tau}<\gamma$. From Corollary 8 , it is obvious that our results improve the results in [12].

\section{Example}

Example 1. We consider the following neutral stochastic partial functional differential equation with impulses:

$$
\begin{aligned}
& d\left[x(t)+a_{1} x(t-1)\right] \\
& =\left[\frac{\partial^{2}}{\partial z^{2}} x(t)+a_{2} x(t-1)\right] d t \\
& \quad+a_{3} x(t-1) d w(t), \quad 0 \leq z \leq \pi, t \geq 0, t \neq t_{k}, \\
& \Delta x\left(t_{k}\right)=I_{k}\left(x\left(t_{k}^{-}\right)\right)=\frac{b_{1}}{k^{2}} x\left(t_{k}^{-}\right), \quad t=t_{k}, \quad k=1,2, \ldots, \\
& x_{0}(s)=\phi(s) \in \mathrm{PC}_{\mathscr{F}_{t}}^{b}\left([-1,0], L^{2}[0, \pi]\right), \\
& \quad x(t, 0)=x(t, \pi)=0, \quad-1 \leq s \leq 0,
\end{aligned}
$$

where $a_{i}>0, i=1,2,3, b_{1} \leq 0$ are constants and $w(t)$ denote the standard cylindrical Wiener process.

Let $H=L^{2}[0, \pi], H_{1}=W^{2,2}(0, \pi) \cap W_{0}^{1,2}(0, \pi)$. Define bounded linear operator $A: H_{1} \rightarrow H$ by

$$
A x=\frac{\partial^{2} x}{\partial z^{2}} \in H, \quad \forall x \in H_{1} .
$$

Then we get

$$
A x=\sum_{n=1}^{\infty} n^{2}\left\langle x, e_{n}\right\rangle_{H} e_{n}, \quad x \in H_{1},
$$


where $e_{n}(z)=\sqrt{2 / \pi} \sin n z, n=1,2, \ldots$ is the set of eigenvector of $-A$.

The bounded linear operator $(-A)^{3 / 4}$ is given by

$$
(-A)^{3 / 4} x=\sum_{n=1}^{\infty} n^{3 / 2}\left\langle x, e_{n}\right\rangle_{H} e_{n}
$$

with domain

$$
D\left((-A)^{3 / 4}\right)=H_{3 / 4}=\left\{x \in H, \sum_{n=1}^{\infty} n^{3 / 2}\left\langle x, e_{n}\right\rangle_{H} e_{n} \in H\right\} .
$$

We can easily know that $A$ is the infinitesimal generator of an analytic semigroup $S(t), t \geq 0$, in $H$ and

$$
S(t) x=\sum_{n=1}^{\infty} e^{-n^{2} t}\left\langle x, e_{n}\right\rangle_{H} e_{n}, \quad x \in H .
$$

Furthermore, we know that $\|S(t)\| \leq e^{-\pi^{2} t}, t \geq 0$.

Let $g(t, x(t-\tau))=a_{1} x(t-1), f(t, x(t-\tau))=a_{2} x(t-1)$, $\sigma(t, x(t-\tau))=a_{3} x(t-1)$, and $t_{k+1}=t_{k}+1$.

Then we can get

$$
\begin{gathered}
M=1, \quad \gamma=\pi^{2}, \quad \tau=1, \quad L_{f}=a_{2}, \\
L_{g}=a_{1}\left\|(-A)^{3 / 4}\right\|, \quad L_{\sigma}=a_{3}, \quad \theta=1, \\
q_{k}=\frac{b_{1}}{k^{2}}, \quad\left\|(-A)^{3 / 4}\right\|=1, \quad\left\|(-A)^{-3 / 4}\right\| \leq \frac{1}{\pi^{3 / 2}} .
\end{gathered}
$$

From Theorem 4, we know that the mild of system (30) is exponentially stable in mean square provided that

$$
\left[\frac{18 M_{1 / 4}^{2} a_{1}^{2}}{\pi^{1 / 2}}+\frac{3 a_{2}^{2}}{\pi^{2}}+3 a_{3}^{2}\right] e^{\pi^{2}}+\ln \frac{\pi^{4}}{6}<\pi^{2}-\frac{18 a_{1}^{2} e^{\pi^{2}}}{\pi} .
$$

\section{Conclusion}

In this paper, neutral stochastic partial functional differential equation with impulses has been investigated. By utilizing impulsive inequality, the stochastic analytic technique, inequality technique, the fractional power of operator, and semigroup theory, some completely new sufficient conditions are obtained to guarantee the exponential stability of mild solution for neutral stochastic partial functional differential equation with impulses. Finally, it should be pointed out that it is possible to extend the results obtained in this paper to other neutral stochastic impulsive differential equations, such as neutral stochastic impulsive partial differential equations with Poisson jumps and so forth.

\section{Acknowledgments}

The author would like to thank the referee and the editor for their careful comments and valuable suggestions on this work. This work is supported by the Key Foundation of Chongqing Three Gorges University (no. 12ZD19).

\section{References}

[1] Y. Ren and R. Sakthivel, "Existence, uniqueness, and stability of mild solutions for second-order neutral stochastic evolution equations with infinite delay and Poisson jumps," Journal of Mathematical Physics, vol. 53, no. 7, Article ID 073517, 14 pages, 2012.

[2] R. Sakthivel, P. Revathi, and S. M. Anthoni, "Existence of pseudo almost automorphic mild solutions to stochastic fractional differential equations," Nonlinear Analysis: Theory, Methods \& Applications, vol. 75, no. 7, pp. 3339-3347, 2012.

[3] R. Sakthivel, P. Revathi, and Y. Ren, "Existence of solutions for nonlinear fractional stochastic differential equations," Nonlinear Analysis: Theory, Methods \& Applications, vol. 81, pp. 70-86, 2013.

[4] R. Sakthivel and Y. Ren, "Exponential stability of second-order stochastic evolution equations with Poisson jumps," Communications in Nonlinear Science and Numerical Simulation, vol. 17, no. 12, pp. 4517-4523, 2012.

[5] R. Sakthivel, Y. Ren, and H. Kim, "Asymptotic stability of second-order neutral stochastic differential equations," Journal of Mathematical Physics, vol. 51, no. 5, Article ID 052701, 9 pages, 2010.

[6] A. Anguraj and A. Vinodkumar, "Existence, uniqueness and stability of random impulsive stochastic partial neutral functional differential equations with infinite delays," Journal of Applied Mathematics information, vol. 28, pp. 739-751, 2010.

[7] A. Anguraj and A. Vinodkumar, "Existence, uniqueness and stability results of random impulsive semilinear differential systems," Nonlinear Analysis: Hybrid Systems, vol. 4, no. 3, pp. 475-483, 2010.

[8] R. Sakthivel and J. Luo, "Asymptotic stability of impulsive stochastic partial differential equations with infinite delays," Journal of Mathematical Analysis and Applications, vol. 356, no. 1, pp. 1-6, 2009.

[9] R. Sakthivel and J. Luo, "Asymptotic stability of nonlinear impulsive stochastic differential equations," Statistics \& Probability Letters, vol. 79, no. 9, pp. 1219-1223, 2009.

[10] J. Luo, "Fixed points and exponential stability of mild solutions of stochastic partial differential equations with delays," Journal of Mathematical Analysis and Applications, vol. 342, no. 2, pp. 753-760, 2008.

[11] J. Luo, "Exponential stability for stochastic neutral partial functional differential equations," Journal of Mathematical Analysis and Applications, vol. 355, no. 1, pp. 414-425, 2009.

[12] T. Caraballo and K. Liu, "Exponential stability of mild solutions of stochastic partial differential equations with delays," Stochastic Analysis and Applications, vol. 17, no. 5, pp. 743-763, 1999.

[13] L. Wan and J. Duan, "Exponential stability of non-autonomous stochastic partial differential equations with finite memory," Statistics \& Probability Letters, vol. 78, no. 5, pp. 490-498, 2008.

[14] H. Chen, "Impulsive-integral inequality and exponential stability for stochastic partial differential equations with delays," Statistics \& Probability Letters, vol. 80, no. 1, pp. 50-56, 2010.

[15] S. Long, L. Teng, and D. Xu, "Global attracting set and stability of stochastic neutral partial functional differential equations with impulses," Statistics \& Probability Letters, vol. 82, no. 9, pp. 1699-1709, 2012.

[16] A. Pazy, Semigroups of Linear Operators and Applications to Partial Differential Equations, vol. 44 of Applied Mathematical Sciences, Springer, New York, NY, USA, 1983. 
[17] A. M. Samoĭlenko and N. A. Perestyuk, Impulsive Differential Equations, vol. 14 of World Scientific Series on Nonlinear Science. Series A: Monographs and Treatises, World Scientific, River Edge, NJ, USA, 1995.

[18] T. Taniguchi, "Almost sure exponential stability for stochastic partial functional-differential equations," Stochastic Analysis and Applications, vol. 16, no. 5, pp. 965-975, 1998. 


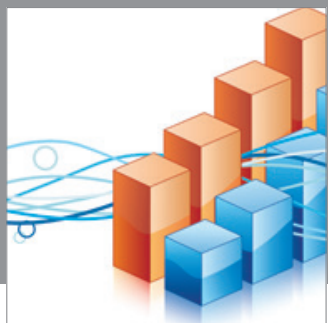

Advances in

Operations Research

mansans

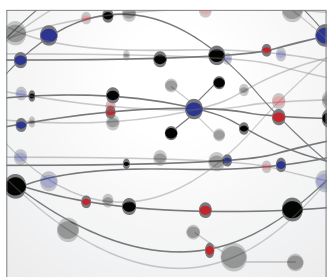

The Scientific World Journal
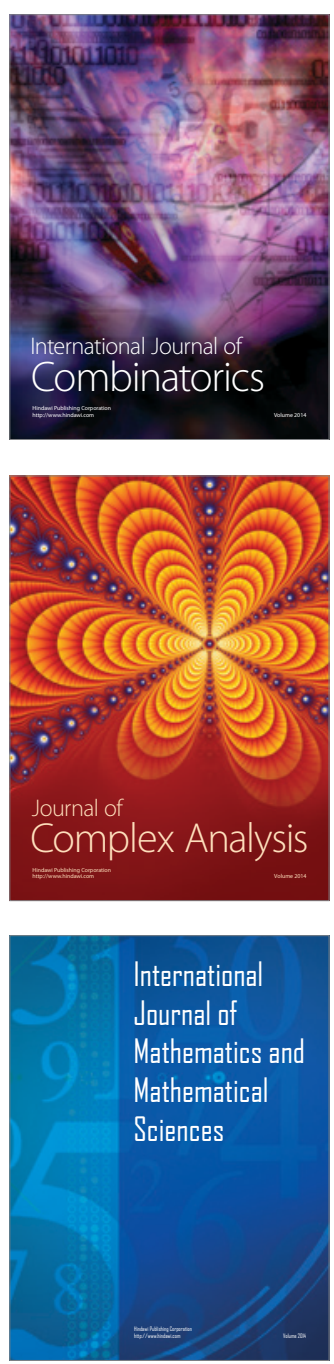
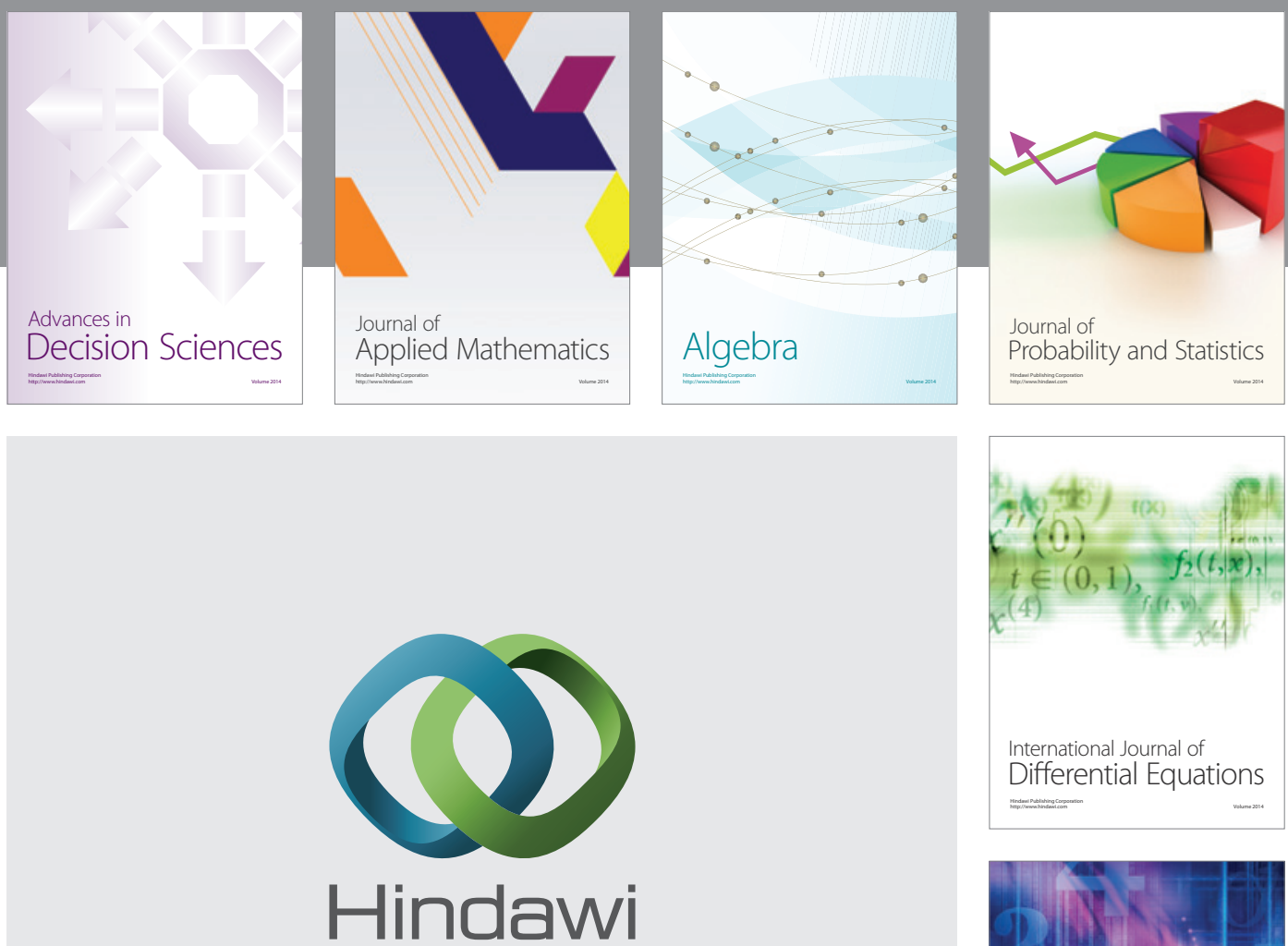

Submit your manuscripts at http://www.hindawi.com
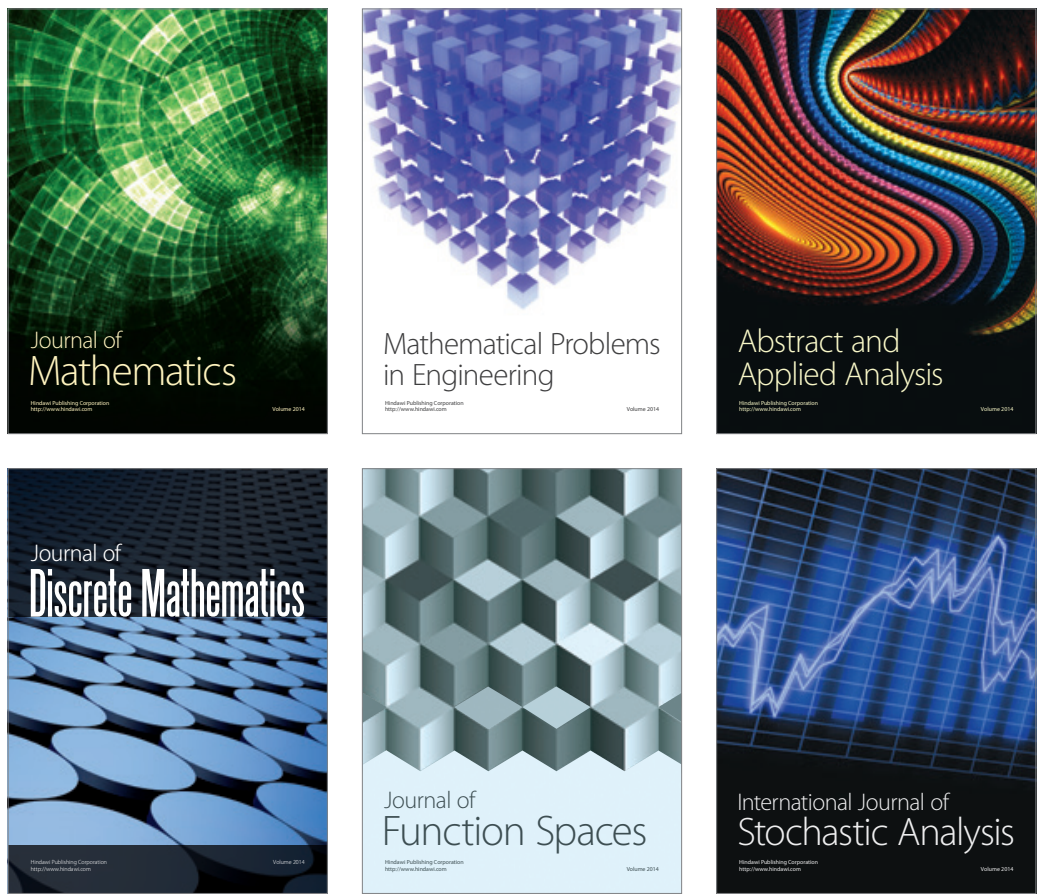

Journal of

Function Spaces

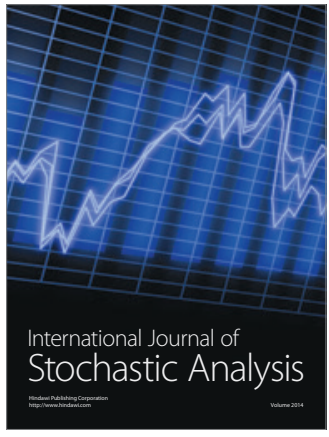

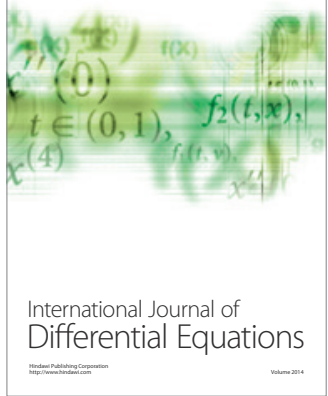
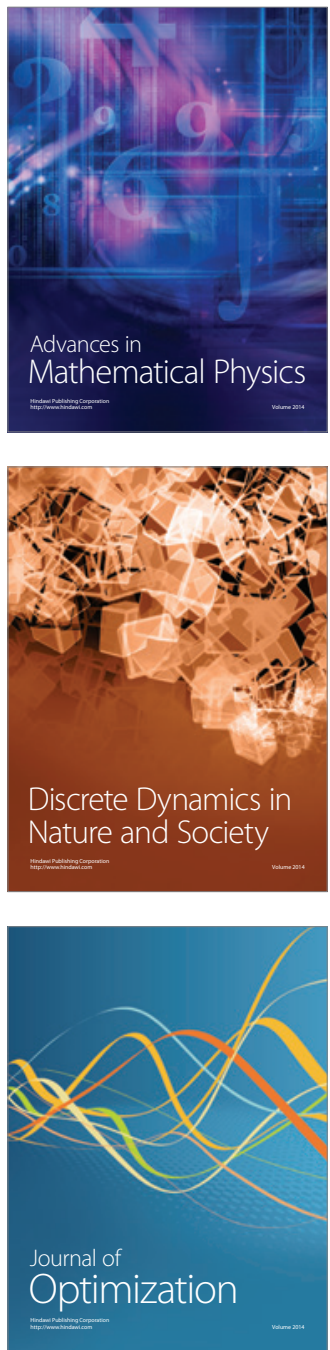